\title{
LA PRODUCCIÓN DEL CONOCIMIENTO EN EDUCACIÓN EN ENFERMERÍA EN ESPAÑA Y BRASIL: UNA REVISIÓN INTEGRATIVA
}

\author{
Marta Lenise do Prado ${ }^{1}$, José Luís Medina-Moya², José Ramón Martínez-Riera ${ }^{3}$
}

${ }^{1}$ RN, PhD. Profesora Departamento de Enfermería y Programa de Pós-grado en Enfermería de la Universidad Federal de Santa Catarina, Brasil. E-mail: mpradop@ccs.ufsc.br

${ }^{2}$ RN, PhD. Profesor Departamento Didáctica y Organización Educativa. Facultad de Pedagogía. Universidad de Barcelona, Espanha. E-mail: jlmedina@ub.edu.es

${ }^{3}$ RN, PhD. Profesor Departamento Enfermería Comunitaria, Medicina Preventiva y Salud Pública e Historia de la Ciencia. Universidad de Alicante, Espanha. E-mail: jr.martinez@ua.es

\begin{abstract}
RESUMEN: El presente estudio es una revisión integrativa con el objetivo de conocer el estado del arte del conocimiento sobre educación en enfermería, en el período de 2000 a 2005, en Brasil y España. Los datos fueron recolectados de las bases CUIDEN (España) y LILACS (Brasil), y analizados estadísticamente (números absolutos). Los artículos fueron clasificados por temas a partir de seis categorías: proceso de enseñanza-aprendizaje, docentes, currículum, evaluación, alumnos, e historia de la educación en enfermería, y, por tipo de trabajo: investigación (cualitativa, cuantitativa e histórica), relato de experiencia, reflexión, editorial y revisión. Los resultados demostraron que hay similitudes y diferencias en la producción científica de Brasil y España. En ambos países son pocos los artículos que tratan del tema de la formación pedagógica de los enfermeros, y la mayoría son artículos de investigación. Con relación a la naturaleza de la investigación, en Brasil se encontró un predominio de investigaciones cualitativas, mientras que en España predominan las investigaciones cuantitativas.
\end{abstract}

DESCRIPTORES: Enfermería. Educación superior. Investigación en enfermería.

\section{NURSING EDUCATION KNOWLEDGE PRODUCTION IN SPAIN AND BRAZIL: AN INTEGRATIVE REVIEW}

\begin{abstract}
The objective of this integrative review is to better understand publications concerning nursing education in Brazil and Spain from 2000-2005. The data was collected from the CUIDEN and LILACS databases and analyzed using simple statistics. The articles were characterized according to central theme into the following six categories: teaching-learning process, faculty, course curriculum, evaluation, students, and the history of nursing education. They were also categorized according to their type of publication: research, experience report, reflection, editorial, and review. The results demonstrate that there are similarities between Brazilian and Spanish scientific production in this area. Neither country shows many articles which deal with the theme of formal pedagogical structures, with the majority of studies discovered constituting research articles. Concerning the nature of the study, the Brazilian studies were predominantly qualitative, while the majority of the Spanish studies were quantitative.
\end{abstract}

DESCRIPTORS: Nursing. Nursing education. Research in nursing.

\section{A PRODUÇÃO DO CONHECIMENTO EM EDUCAÇÃO EM ENFERMAGEM NA ESPANHA E NO BRASIL: UMA REVISÃO INTEGRATIVA}

\begin{abstract}
RESUMO: Revisão sistemática com o objetivo de conhecer o estado da arte das publicações acerca da educação em enfermagem no período 2000-2005, no Brasil e na Espanha. Os dados foram coletados das bases de dados CUIDEN (Espanha) e LILACS (Brasil) e analisados por meio de estatística simples (número absoluto). Os artigos foram caracterizados por tema central em seis categorias: processo de ensino-aprendizagem, docentes, currículo, avaliação, alunos e historia da educação em enfermagem e por tipo de publicação em: pesquisa (qualitativa, quantitativa e histórica), relato de experiência, reflexão, editorial e revisão. Os resultados demonstraram que há semelhanças e diferenças na produção científica entre Brasil e Espanha. Em ambos os países são poucos os artigos que tratam da temática de formação pedagógica e a maioria deles constituem-se em artigos de pesquisa. Em relação à natureza da pesquisa, no Brasil foi encontrada predominância de estudos qualitativos, enquanto que na Espanha, foram de pesquisas quantitativas.
\end{abstract}

DESCRITORES: Enfermagem. Educação superior. Pesquisa em enfermagem. 


\section{INTRODUCCIÓN}

Desde finales del Siglo XX e inicio del presente siglo se ha producido una aceleración de las transformaciones en el mundo del trabajo que han generado nuevos retos a la formación de los profesionales, especialmente, de los profesionales del área de la salud. Para diversos autores, ${ }^{1-3}$ las exigencias de capacitación, actualización y formación, también han determinado transformaciones en el mundo académico y en los procesos de formación por él desarrollados.

En ese proceso de cambios profundos, tanto Brasil como España se encuentran en plena reestructuración del sistema universitario, iniciada hacia finales de la década de los noventa, afectando también a la educación de enfermería. En España, a partir de 1999, con la Declaración de Bolonia, el sistema educación de la educación superior ha sufrido un proceso de reorientación, que ha determinado un importante reto en su estructura y funcionamiento. La "Declaración de Bolonia, del 19 de junio de 1999, ha supuesto el inicio de una reflexión sobre el papel que debe desempeñar la universidad europea en el desarrollo de la sociedad del conocimiento y bienestar." ${ }^{4: 19}$

En España, hasta recientemente, dicho proceso se ha centrado sobre todo en la realización de cambios en la estructura académica y la organización curricular y no tanto en la innovación pedagógica y el desarrollo de los nuevos enfoques metodológicos que deben acompañar a la nueva organización de las enseñanzas. Se impone pues aprovechar este momento para poner en valor la docencia como profesión y renovar las metodologías educacionales..$^{4-5} \mathrm{~L}$ a educación en enfermería así como todo el sistema educativo, vive momentos de cambio e inicia procesos de discusión e implementación de nuevas experiencias educativas. ${ }^{6} \mathrm{Sin}$ embargo, la producción científica de enfermería se encuentra aún en un estado incipiente debido a su reciente inserción en el sistema de educación superior, y en consecuencia, en el acceso a la formación avanzada, a nivel de doctorado. ${ }^{7}$

En Brasil, la trayectoria de la formación profesional en Enfermería presenta algunas similitudes y diferencias en relación a España. Al igual que España (con el Espacio Europeo de Educación Superior (EEES), 1999), el sistema educativo brasileño se reorganiza según la Ley de Directrices y Bases (LDB) de 1996, de la cual emanan las Directrices Curriculares Nacionales (DCNs), que explicitan las bases filosóficas, conceptuales, po- líticas y metodológicas que, a su vez, definen un conjunto de habilidades y competencias de un área del conocimiento. ${ }^{8}$ Las DCNs proponen una nueva estructura curricular de grado altamente flexible y más allá del modelo curricular cuyo énfasis recaía en la asignatura y en su carga horaria. ${ }^{9}$

En Brasil, desde el inicio de la década de noventa, al contrario de lo que sucedió en España, el sistema de postgrado en enfermería (maestría y doctorado) ha venido acompañando todo el sistema brasileño de postgrado, creciendo de manera significativa. Esto fue fortalecido sobre todo por una de las determinaciones establecidas en la LDB, que establecía que un tercio de los profesores de las universidades debían poseer el título de máster o doctorado. Con ese se produjo un aumento en la demanda de profesores calificados en todos los ámbitos disciplinares, incluida la enfermería. En consecuencia, la creación de grupos de investigación ganó impulso, generando un fuerte aumento de la producción científica en el área. ${ }^{10-11}$

Aunque sea posible identificar los avances en la formación universitaria de Enfermería, tanto en Brasil como en España, cuando esta ingresa en el ámbito universitario adopta, sin visión crítica, los modelos epistemológicos y los valores culturales imperantes en la institución universitaria, la cual está totalmente determinada por la visión previa del docente y centrada en la reproducción de su conocimiento profesional, enfatizando en el contenido como un fin en sí mismo. ${ }^{12-14}$

Todavía hoy, la formación de las enfermeras debe estar en sintonía con los desafíos de la atención a la salud, especialmente, en lo referente a la atención de los principios de autonomía y libertad que deben orientar las prácticas sanitarias. Es necesario superar la fragmentación de la práctica profesional y educativa, la cual debe ser ejercida a través de un proceso de acción-reflexión cooperativo, de indagación y experimentación, en el cual, el profesional se coloque como un eterno aprendiz, donde el diálogo y el respeto son fundamentos de las relaciones interpersonales. ${ }^{15}$

Los avances y los cambios en el desarrollo de los programas de formación profesional en enfermería, las nuevas tendencias e innovaciones educativas, además de los imperativos legales, han sido también producto de diferentes procesos investigativos en el ámbito de la educación en enfermería, tanto en Brasil como en España. Un estudio comparado sobre la producción del conocimiento en educación en enfermería, en 
Brasil y España - dos países que en el contexto de la educación en enfermería viven procesos de cambios educativos semejantes, puede enriquecer el intercambio de experiencias, favorecer la cooperación internacional y fomentar actividades investigativas conjuntas. Por lo tanto, el presente estudio tiene como objetivo conocer el estado del arte del conocimiento acerca de la educación en enfermería en el período de 2000 a 2005, en Brasil y España.

\section{METODOLOGÍA}

En el presente estudio se realiza una revisión integrativa ${ }^{16}$ de las publicaciones sobre la educación en enfermería, en Brasil y España, en el período de 2000 a 2005. Los datos referentes a España fueron recolectados de la base de datos CUIDEN, por ser una base que engloba un amplio universo de revistas de enfermería publicadas en Iberoamérica, las cuales son representativas de las revistas utilizadas por los enfermeros españoles para sus publicaciones. La estrategia de búsqueda en esa base de datos fue la siguiente: en el lugar "Búsqueda avanzada" se colocó el término educación en enfermería (palabra clave); para la fecha, 2000 (el año); y artículo, para el tipo de publicación, empleando ese mismo método para cada año.

Los datos relativos a Brasil fueron obtenidos de la base LILACS, por tratarse de una base latinoamericana que abarca la mayoría de las revistas brasileñas y latinoamericanas, donde se concentra gran parte de las publicaciones de los enfermeros brasileños. La búsqueda fue hecha con la siguiente estrategia: En el lugar "formulario básico" se utilizó el término educación en enfermería (descriptor de asunto); Brasil - 2000 (país y año), así sucesivamente para cada año. Fue considerada como publicación del país, aquélla cuyos autores se encuentran vinculados a instituciones de Brasil o España, y/o sus estudios fueron realizados en esos países.

A partir de una primera lista se procedió a realizar una lectura de cada uno de los resúmenes de los artículos, identificando los que correspondían a la temática de este estudio, y descartando los artículos que abordaban el tema desde otra perspectiva, tales como educación continuada y educación en salud. También fueron descartados los estudios publicados por enfermeras de otros países, comunicaciones y tesis, así como los artículos que estaban duplicados. Después de una primera lectura, los resúmenes seleccionados formaron el banco de datos por año y país. En la siguiente etapa, los resúmenes fueron clasificados por tema central y tipo de publicación. El tema central es el foco principal del artículo y fue dividido en seis categorías: Proceso de enseñanzaaprendizaje (estudios relativos a las metodologías y tecnologías educativas, a los nuevos modelos de enseñanza); Docente (estudios acerca de la formación de docentes de enfermería, actitudes y creencias de los docentes, perfil); Curriculum (artículos que abordaban las cuestiones relativas a la estructura y organización curricular, contenidos, modelos curriculares, evaluación del plan curricular); Evaluación (estudios que trataban sobre cuestiones relacionadas con el contexto político-estructural de la educación en enfermería, las transformaciones sociales y su impacto en la educación, los retos y desafíos para la educación en enfermería); Alumnos (estudios que tratan de creencias, actitudes, perfil, sentimientos de los alumnos); Historia de Enfermería (estudios acerca de la historia de la educación en enfermería).

Por tipo de publicación, los resúmenes fueron clasificados en: investigación (cualitativa, cuantitativa, histórica), relato de experiencia, reflexión, editorial y revisión. Cuando el resumen no contenía las informaciones necesarias para su clasificación, se realizó la búsqueda del texto completo para proceder a su clasificación. Si el texto no fue localizado o no estaba disponible, fue clasificado como sin información (un estudio en España y 25, en Brasil). La búsqueda fue realizada en mayo de 2009. El banco final se formó con un total de 81 publicaciones de enfermeras(os) españolas(es) y 232 enfermeras(os) brasileñas(os), relacionadas con la temática de educación en enfermería.

\section{RESULTADOS Y DISCUSIÓN}

La educación en enfermería comienza a fortalecerse en el seno disciplinar a partir de las reformas educativas desencadenadas en ambos países a finales de la década de noventa. Las demandas de reestructuración curricular y de cambios metodológicos llevan a los profesionales de enfermería, y en especial, a los involucrados con la enseñanza de enfermería, a una creciente preocupación con las cuestiones didáctico-pedagógicas. Con una significativa producción investigativa dirigida a las prácticas clínicas, la temática de la formación/ educación en enfermería siempre ha sido periférica, tal como lo demuestran algunos estudios. ${ }^{17-18}$ 
Entre esos estudios se puede citar uno realizado en América Latina ${ }^{19}$, basado en los aportes de seis Coloquios Panamericanos de Investigación en Enfermería realizados en la década 1988-1998, donde se encontró que únicamente el 18,7\% de los trabajos presentados exploraron la temática de recursos humanos con énfasis en la formación de enfermería. Se concluye que en general, con excepción de Brasil, los estudios no estaban vinculados a líneas o grupos de investigación, demostrando insuficiencia en la institucionalización de la investigación de educación en enfermería, $y$, por lo tanto, continúa siendo un trabajo marginal en relación a las funciones administrativas, asistenciales y de enseñanza.

Otro estudio acerca de la producción del conocimiento en enfermería en América Latina ${ }^{20}$, en el año 2000, demostró que las líneas de investigación que tratan sobre el tema de las políticas y prácticas de educación y enfermería son las que presentan un mayor número de investigaciones, seguidas por la línea de producción en salud y trabajo de enfermería, con pocos estudios sobre la formación de recursos humanos, evaluación educacional, caracterización de los alumnos, docentes y política educacional en enfermería. Ese hecho revela una tendencia de investigaciones en los aspectos organizacionales e históricos del trabajo de enfermería, con destaque en la gestión, y una inexpresiva investigación en el área de educación.

En Brasil y en España, la preocupación por la temática educación en enfermería se manifiesta de modo creciente al comienzo del siglo XXI. Ese aumento parece estar relacionado con los movimientos generales de las políticas educacionales ocurridas en ambos países. En Brasil, con la publicación de la LDB, y en España, con la implementación del EEES. Ambos eventos son paradigmáticos de la movilización de las instituciones de educación superior, ya que indicaban cambios significativos en el modelo de educación superior. Las escuelas de enfermería, los profesionales y las organizaciones profesionales comienzan a preocuparse con la temática de la formación inicial de manera más significativa, para dar respuestas a las exigencias de adecuación de sus modelos educativos.

La anterior situación puede ser percibida en la presente revisión sistemática, en el período de 2000 a 2005. Las publicaciones acerca del tema educación en enfermería, en Brasil y en España, presentan una distribución bastante irregular a lo largo del período. En España, en el año de 2003, se percibe un aumento que es seguido de un des- censo en los años siguientes, sin embargo, hay una producción expresiva en el año 2005. En Brasil, un número mayor de publicaciones en educación en enfermería se presenta en el año de 2003 (Figura 1).

Figura 1 - El total de publicaciones en educación en enfermería, disponibles en las bases de datos LILACS (Brasil) y CUIDEN (España), 2000-2005

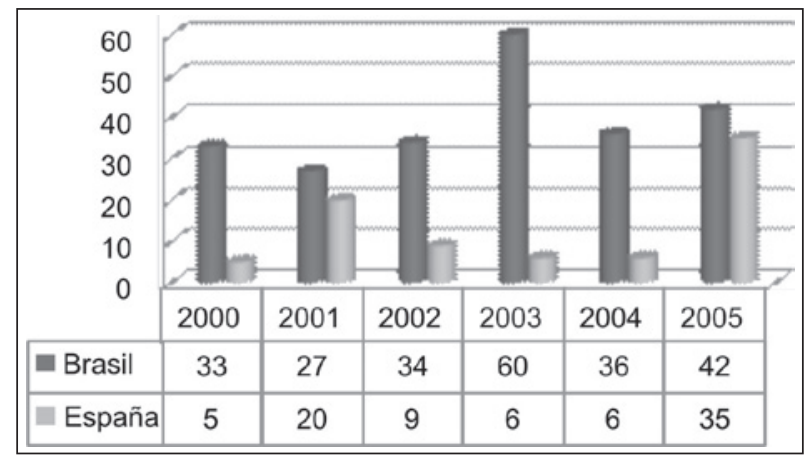

Este estudio muestra que además de ser la educación una preocupación creciente en la producción del conocimiento en enfermería, las temáticas más abordadas en las publicaciones, tanto en España como en Brasil, son las que corresponden al proceso enseñanza-aprendizaje, el currículum, evaluación del contexto y alumnos (Figuras 2 y 3). Llama la atención el hecho de que la categoría docentes sea la que presenta un menor número de estudios en ambos países. Los estudios acerca de los docentes son una cuestión central en la educación, ya que es uno de los pilares en el proceso enseñanza-aprendizaje. Sin embargo, la formación pedagógica del docente de enfermería aún es un tema que ha movilizado poco a los investigadores enfermeros.

Figura 2 - Distribución de la publicación en educación en Enfermería, por tema central. España, 2000-2005

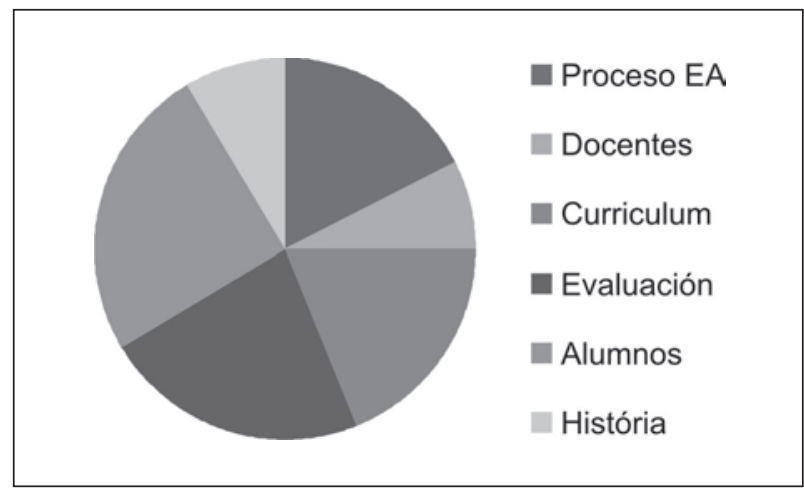


Figura 3 - Distribución de la publicación en educación en Enfermería, por tema central. Brasil, 2000-2005

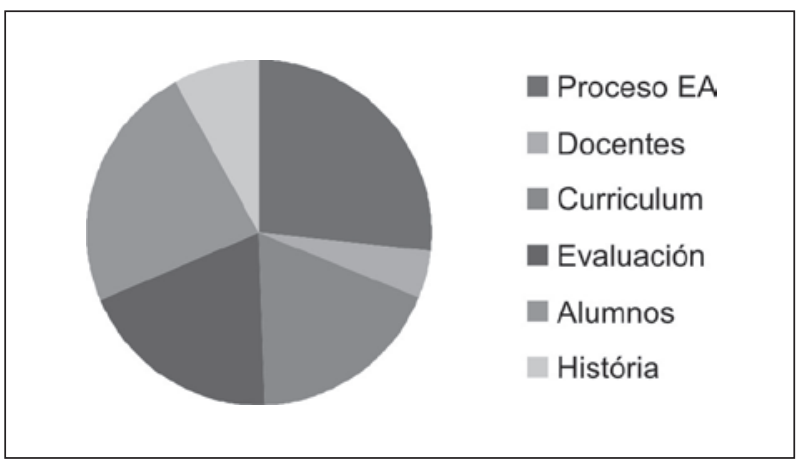

Los datos del presente estudio demuestran que las temáticas todavía se distribuyen de manera diferente a lo largo del período de estudio. En Brasil, por ejemplo, la categoría Evaluación del contexto presenta una fuerte manifestación en 2003, mientras que en España, aparece con fuerte tendencia en 2005 (Tablas 1 y 2). Con ese hecho se puede demostrar el creciente proceso de movilización y la preocupación de los enfermeros con los contextos más generales de la educación y su reflejo en la educación de enfermería.

Tabla 1 - Distribución de la producción científica sobre educación en enfermería, de acuerdo con la temática central y año. LILACS, Brasil, 2000-2005

\begin{tabular}{lccccccc}
\hline \multicolumn{1}{c}{ Ano/Categoria } & $\begin{array}{c}\text { Processo } \\
\text { EA }\end{array}$ & Docente & Currículum Evaluacion & Alumno & História & Total \\
\hline 2000 & 12 & 01 & 08 & 01 & 08 & 03 & 33 \\
2001 & 04 & 03 & 10 & 01 & 07 & 02 & 27 \\
2002 & 09 & 01 & 05 & 09 & 06 & 04 & 34 \\
2003 & 10 & - & 12 & 19 & 12 & 05 & 58 \\
2004 & 11 & 05 & 02 & 06 & 11 & 03 & 38 \\
2005 & 16 & - & 06 & 08 & 10 & 02 & 42 \\
\hline Total & $\mathbf{6 2}$ & $\mathbf{1 0}$ & $\mathbf{4 3}$ & $\mathbf{4 4}$ & $\mathbf{5 4}$ & $\mathbf{1 9}$ & $\mathbf{2 3 2}$ \\
\hline
\end{tabular}

Tabla 2 - Distribución de la producción científica sobre educación en enfermería de acuerdo con la temática central y año. CUIDEN, España, 2000-2005

\begin{tabular}{lccccccc}
\hline \multicolumn{1}{c}{ Ano/Categoria } & $\begin{array}{c}\text { Processo } \\
\text { EA }\end{array}$ & Docente & Currículum Evaluacion & Alumno & História & Total \\
\hline 2000 & 01 & - & 01 & - & 02 & 01 & 05 \\
2001 & 07 & 04 & 04 & - & 04 & 01 & 20 \\
2002 & - & 01 & 01 & - & 04 & 03 & 09 \\
2003 & - & - & 01 & - & 04 & 01 & 06 \\
2004 & - & - & 02 & 02 & 02 & - & 06 \\
2005 & 06 & 01 & 06 & 16 & 04 & 01 & 34 \\
\hline Total & $\mathbf{1 4}$ & $\mathbf{0 6}$ & $\mathbf{1 5}$ & $\mathbf{1 8}$ & $\mathbf{2 0}$ & $\mathbf{0 7}$ & $\mathbf{8 0}$ \\
\hline
\end{tabular}

Ese hecho es corroborado por la constatación de que los estudios sobre evaluación son en general reflexiones (14 de los 18 estudios publicados en España y 38 de los 44 en Brasil), y están relacionadas con el contexto de cambio en el sector educativo en que están involucrados ambos países. En España aparecen, entre otras, las siguientes temáticas: Tuning y la enfermería, reglamentación de los estudios de postgrado, realidad global e implicaciones para la educación en enfermería, inmigración y formación transcultural, nueva ordenación universitaria, escenario 
futuro de formación, integración de los estudios de enfermería en ESSE, formación en enfermería y la coyuntura europea.

En Brasil, las temáticas también se refieren al contexto político educativo nacional, los nuevos paradigmas educativos y las repercusiones de los cambios en los procesos educativos. Así aparecen los siguientes temas centrales: integralidad, interdisciplinaridad y currículum, creencias epistemológicas en el aprendizaje de enfermería, desarrollo sostenido, construcción del conocimiento en la enseñanza de enfermería, educación reflexiva en la postmodernidad, educación tecnológica y salud, complejidad y formación, modelo de competencias y los cambios en el mundo de trabajo, ciudadanía y ética, directrices curriculares y formación, cambio en los abordajes pedagógicos.

No sólo en España y Brasil han ocurrido procesos que han generado debates en relación a la temática de educación en enfermería. El interés sobre esa temática se constata en otros países, los cuales también están sufriendo cambios en sus sistemas educativos. Un estudio realizado en Colombia acerca del contexto de la producción investigativa en educación en enfermería (1995-2004), demostró que las áreas temáticas más exploradas son: la competencia comunicativa y su relación con la construcción del conocimiento, las pedagogías, los modelos pedagógicos, la didáctica, el proceso de enseñanza aprendizaje. Y sólo después de la década de los noventa son tratados otros temas, tales como: educación y salud, la evaluación, los currículos, la formación de maestros y la enseñanza de las asignaturas. En ese estudio también parece que el impulso de la investigación en educación en enfermería (y en especial, de algunas temáticas,) se hace más visible después de los cambios en los modelos pedagógicos que ocurren de manera más destacada durante la década de los noventa. ${ }^{21}$

Otro estudio sobre la producción científica de Enfermería en España, en el período de 1998-2002, reveló que el área de conocimiento más estudiada ha sido la Enfermería médico-quirúrgica, seguida de la Enfermería comunitaria. En el análisis temporal se observó un incremento de artículos en las áreas Materno-infantil, Salud Mental y Geriatría y Gerontología, así como un descenso en los artículos de Gestión. ${ }^{22}$ Sin embargo, no es relevante la temática de educación en enfermería, lo que indica que en ese período las publicaciones acerca de esa temática aún eran escasas o tenían poco interés.

Estos resultados difieren de los encontrados en un estudio ${ }^{23}$ sobre la tendencia de las investiga- ciones desarrolladas por la enfermería en el ámbito internacional, cuyos datos fueron obtenidos de dos revistas de enfermería, (Nursing and Health Sciences $y$ del Journal of Research in Nursings, publicados en el período de 2005-2006), donde el área temática más investigada fue la formación profesional $(19,2 \%)$, seguida de la salud del adulto $(11 \%)$, salud colectiva $(11 \%)$ y cuidado y autonomía $(9,6 \%)$.

Otro dato a destacar es el relativo a la naturaleza de las publicaciones de educación en enfermería. La mayoría de los artículos publicados en Brasil (45,6\%) y en España (33,7\%) son sobre investigaciones, seguido por los trabajos de reflexión (26,2\% en Brasil y $28,7 \%$ en España) y relatos de experiencia (23,7\% en Brasil y 18,7\% en España). Es un dato semejante al encontrado en un estudio sobre la producción científica de Enfermería en América Latina (1959-2005), en que el 69\% de las publicaciones eran de artículos originales, o sea, comunicación de resultados de investigación ${ }^{24}$. En ambos países (Brasil - 1\% y España - 6,2\%) aún se identifica un pequeño número de editoriales acerca de esa temática. Ese es un hecho que llama la atención pues a pesar de parecer un tema de importancia creciente, no se ve reflejado en los editoriales de las revistas científicas de enfermería.

En otro estudio ${ }^{22}$ realizado sobre las publicaciones en España (1998-2002) se demostró que la mayoría de los trabajos eran de aplicación tecnológica, seguido de los clasificados como artículos de opinión. Solamente el 21,6\% son artículos de investigación, resultado que diverge del encontrado en el presente estudio, demostrando que hubo un incremento de los artículos de investigación, lo que sugiere el fortalecimiento de la actividad investigativa en el período 2000-2005, no solamente en la temática de educación, sino en el contexto general de la producción del conocimiento en Enfermería. Es necesario reconocer que el incremento de la calidad editorial de las revistas de enfermería ha exigido un mayor número de publicaciones de artículos de investigación, ya que es uno de los criterios de valoración establecidos por la comunidad editorial para las revistas científicas.

La mayor diferencia observada en las investigaciones producidas en los dos países es relativa al diseño. En España se destacan los diseños cuantitativos (85\%) y en Brasil, los cualitativos (60\%). En España, los diseños cuantitativos aparecen en 23 de las 27 investigaciones publicadas, siendo los más frecuentes los descriptivos (transversal, correlacional, prospectivo). Los diseños cualitativos (7,5\%) consistieron en un grupo de discusión y un estudio 
de observación. También fueron encontrados dos estudios históricos (7,5\%). En Brasil, los diseños cualitativos son identificados como: investigación convergente-asistencial, investigación-acción, fenomenología, etnometodología, análisis de contenido, estudio de caso, teoría fundamentada en los datos, estudio exploratorio, representaciones sociales, investigación participante, $\mathrm{y}$, algunos que solamente son identificados como de naturaza cualitativa. Con relación a los estudios cuantitativos (28 estudios - 30\%), los diseños encontrados son frecuentemente exploratorio-descriptivos, aunque aparecen algunos estudios correccionales (3) y experimental (1). También fueron identificadas 10 publicaciones (10\%) como estudios históricos, y seis estudios en los cuales no fue posible obtener información acerca del diseño.

En uno estudio ${ }^{21}$ también se identificó el uso de abordajes cualitativos y cuantitativos en los estudios realizados en Colombia. Según las autoras, las metodologías utilizadas van desde la investigación descriptiva y cuantitativa pasando por diseños de investigación cualitativa (la etnográfica, la hermenéutica, la investigación de carácter evaluativo).

De modo general, en España y Brasil, la distinción de los diseños utilizados en las investigaciones observadas se relaciona con el estado de la investigación en cada uno de los países. En Brasil, hay un predominio de estudios cualitativos en el área de Enfermería, mientras que en España se observa una mayoría de estudios de naturaleza cuantitativa, comportamiento que también es visible en otros países del mundo.

Un estudio ${ }^{24}$ realizado en el período de 20052006, demostró que así como en Brasil, la mayoría de las investigaciones publicadas en dos periódicos norteamericanos (Nursing and Health Sciences y Journal of Research in Nursings) fueron de abordajes cualitativos (53,9\%), y a la vez, las investigaciones con diseño cuantitativo representaron el 46,1\% de las publicaciones.

Resultados diferentes se encontraron en otro estudio acerca de las tendencias internacionales de la investigación en enfermería (2005-2006). Las autoras $^{25}$ encontraron un predominio de estudios cuantitativos o cuantitativos-cualitativos $(63.8 \%)$, siendo los estudios cualitativos mayoritarios solamente en algunos países de Europa como Noruega, Suecia y Reino Unido. Dentro de los estudios cuantitativos, los descriptivos-exploratorios fueron los más frecuentes, ya que solamente el 13,9\% eran estudios de intervención (experimental o casi experimental).

\section{CONSIDERACIONES FINALES}

Los datos presentados en este estudio ponen de manifiesto una realidad de importantes coincidencias y diferencias en la producción del conocimiento en el área de la educación en enfermería. La coincidencia más significativa se encuentra en el escaso número de estudios acerca de los docentes, especialmente, en lo que se refiere a la formación pedagógica de las enfermeras. Ese hecho se destaca como un vacío importante en los estudios investigativos en el área, además de demostrar que aún no es un tema considerado de relevancia para la enfermería. Es ese sentido, se hace necesaria una revisión de esa situación, ya que la formación pedagógica de las enfermeras debe, además de los otros temas, estar en las pautas investigativas del área, pues el docente tiene un papel central en la implementación de los procesos de cambio que están ocurriendo en Brasil y en España.

La educación en enfermería ha evolucionado de una postura educativa más impositiva hacia una actitud más reflexiva. Con ello se ha podido salir de un modelo de atención basado en el paradigma biomédico, con alto nivel técnico-mecanicista, hacia el paradigma holístico-disciplinario, más cercano a la Enfermería. Ese cambio paradigmático ha permitido capacitar a las enfermeras mediante la adquisición de las aptitudes y competencias necesarias para su incorporación inmediata al mercado laboral y para estimular el desarrollo de la ciencia y el espíritu de reflexión, el fortalecimiento de la profesión y el desarrollo de la investigación en enfermería. ${ }^{26-27}$

“Enseñar a aprender, más que la mera transmisión de contenidos será el principal objetivo del profesor, determinado por el paso de instructor a guía, capaz de proporcionar ayuda y apoyo al estudiante. Orientador para potenciar que el estudiante forme parte activa del proceso de aprendizaje. Posibilitador y motivador para asesorar y gestionar el ambiente de aprendizaje, el uso de las bases de la información y del conocimiento". $28: 158$ Hacer ese cambio de rol requiere el desarrollo de competencias pedagógicas que capaciten a las enfermeras a comprender los fundamentos ontológicos y epistemológicos del conocimiento pedagógico y sus implicaciones para la formación profesional. Además, los cambios curriculares que se hacen necesarios (y que están en marcha), tanto en Brasil como en España, exigen de los docentes enfermeros una nueva comprensión de la relación teoría-práctica en el currículum de enfermería y la implementación de la enseñanza del cuidado como 
una práctica reflexiva ${ }^{29}$, lo que exige un permanente proceso de reflexión-acción-reflexión sobre las prácticas pedagógicas. Prácticas pedagógicas que deben estar ancladas en la realidad del cuidado en salud, y desarrolladas teniendo como norte un proyecto pedagógico consciente (o sea, un rol fundamental del profesor), para contribuir con las transformaciones no sólo en el espacio educativo, sino también en los servicios de salud.

La diferenciación de abordajes de investigación (cuantitativo y cualitativo) predominantes en ambos países es otra característica a ser destacada, ya que pude ser una fortaleza para la implementación de intercambios y estudios multicéntricos. Abordajes diferentes permiten miradas distintas acerca del fenómeno educativo en enfermería y permite vislumbrar diferentes perspectivas de acción ante la complejidad de las prácticas educativas como las de salud.

A partir del presente estudio es posible percibir oportunidades para el desarrollo investigativo de enfermería, con énfasis en la formación de líderes que puedan actuar en los nuevos cambios educativos y de salud, así como la formación de investigadores capaces de promover un impacto en la construcción de nuevos conocimientos científicos y tecnológicos para todos los países de Iberoamérica. El reconocimiento del estado del arte de la investigación de educación en enfermería, ayuda en la identificación de políticas para la educación y nuevos enfoques en la investigación, con el objetivo de contribuir al desarrollo de la formación profesional de enfermería en la realidad iberoamericana.

\section{REFERENCIAS}

1. Delors J. Educação um tesouro a descobrir: relatório para a UNESCO da comissão internacional sobre educação para o século XXI. $4^{\mathrm{a}}$ ed. São Paulo (SP)/ Brasília(DF): Cortez/MEC/UNESCO; 2000.

2. Haddad AE, Pierantoni CR, Ristoff D, Xavier IM, Giolo J, Silva LB, organizadores. A trajetória dos cursos de graduação na área da saúde: 1991-2004. Brasília (DF): Instituto Nacional de Estudos e Pesquisas Educacionais Anísio Teixeira; 2006.

3. Vasconcellos EM, organizador. Perplexidade na universidade: vivências nos cursos de saúde. São Paulo (SP): HUCITEC/Edições Mandacaru; 2006.

4. Ministerio de Educación y Ciencia (España). Secretaría de Estado de Universidades e Investigación. Propuestas para la renovación de las metodologías educativas en la Universidad, España. 2006 [acceso 2008 Abr 21]. Disponible en: http:/ / www.educacion.es/dctm/mepsyd/educacion/ universidades/estadisticas-informes/estadisticas/ propuestarenovacion.pdf?documentId $=0901 \mathrm{e} 72 \mathrm{~b}$ 80048 b70.

5. Martínez RJR, Cibanal JL, Pérez MMJ. Experiencia docente en el marco del Espacio Europeo de Educación Superior. Metas de Enferm. 2007; 10(3):57-62

6. Martínez RJR, Cibanal JL, Pérez MMJ. Integración docente en el proceso de enseñaza-aprendizaje. Metas de Enferm. 2009; 12(6):50-5.

7. Camaño PR. The state of the nursing science in Spain. Rev Latino-am Enfermagem [online]. 2002 Abr [acceso 2009 Jul 02]; 10(2):214-20. Disponible en: http:/ / www.scielo.br/scielo.php?script=sci_ arttext\&pid=S0104-11692002000200014\&lng=pt.

8. Almeida M.J. Diretrizes curriculares nacionais para os cursos universitários da área da saúde. Londrina (PR): Rede Unida; 2005.

9. Garbin CAS, Saiba NA, Moimoz SAS, Santos KT. O papel das universidades na formação de profissionais na área de saúde. Revista ABENO. 2006 Jan-Jun; 6(1):6-10.

10. Rodrigues RP, Erdmann AL, Silva IA, Fernandes JD, Santos RS, Araújo TJ, et al. A formação de doutores em Enfermagem no Brasil. Texto Contexto Enferm. 2002 Maio-Ago; 11(3):66-76.

11. Rodrigues RAP, Erdmann AL, Silva IA, Fernandes JD, Araújo TL, Vianna LAC, et al. Doctoral education in nursing in Brazil. Rev Latino-am Enfermagem. 2008 Jul-Ago; 16(4):665-71.

12. Medina-Moya JL. Introducción. In: Ferrer V, MedinaMoya JL, Lloret C. La complejidad en Enfermería: Profesión, Gestión, Formación. Barcelona: Laertes; 2003.

13. Medina-Moya JL, Sandín-Esteban MP. La complejidad del cuidado y el cuidado de la complejidad: un tránsito pedagógico de los reduccionismos fragmentantes a las lógicas no lineales de la complejidad. Texto Contexto Enferm. 2006 Jun, 15(2):312-9.

14. Medina-Moya JL, Castillo-Parra S. La enseñanza de la enfermería como una práctica reflexiva. Texto Contexto Enferm. 2006 Jun; 15(2):303-11.

15. Reibnitz K, Prado ML. Inovação e educação em Enfermagem. Florianópolis (SC): Cidade Futura; 2006.

16. Gagnon LH. Integrative reviews of nursing. Rev. Nurs Health,1987; 10 (1):1-11.

17. Hidalgo PPL, Rodríguez AR; Fernández GFP, Medina LIM. Producción científica de los docentes de escuelas universitarias de enfermería andaluzas (1997-1999). Metas Enferm. 2001; 4(40):32-7.

18. Castrillón Agudelo MC. Trends and priorities in nursing research. Rev Latino-am Enfermagem [online]. 2004 Aug [cited 2009 July 01]; 12(4):58388. Available from: http://www.scielo. 
$\mathrm{br} /$ scielo.php? script $=$ sci_arttext\&pid=S0104$11692004000400002 \& \operatorname{lng}=\mathrm{en}$.

19. Ailinger RL, Najera RM, Castrillón MC, Manfredi M. Nursing research in Latin America: 1988-1998. Rev Latino-am. Enfermagem [online]. $2005 \mathrm{Dez}$ [citado 2009 Jul 02];13(6):925-8. Disponible en: http:/ / www. scielo.br/scielo.php?script=sci_arttext\&pid=S0104$11692005000600002 \& \operatorname{lng}=\mathrm{pt}$.

20. Prado ML do, Gelbcke FL. Produção do conhecimento em enfermagem no Brasil: as temáticas de investigação. Rev Bras Enferm. 2001; 54(1):34-42.

21. Jimenéz MA, Gómez SC, Vázquez M, Munar OC. Contexto de la producción investigativa en educación en Enfermería en Colombia 1995-2004. Avances en Enfermería. 2005 Jan-Jun; 23(1):5-17.

22. Serrano GP, Giménez Maroto AM, Arroyo Gordo MdelP. Análisis de la producción científica publicada en la revista Metas de Enfermería. Index Enferm [online]. 2005 Jun [citado 2009 Jun 26]; 14(48-49):78-82. Disponible en: http://scielo. isciii.es $/$ scielo.php? script $=$ sci_arttext\&pid=S113212962005000100021\&lng

23. Silva LMS, Oliveira NRN, Frota MA, Fialho AVM. Pesquisa internacional em enfermagem: tendências temáticas e metodológicas. Rev Bras Enferm [online]. 2008 Oct [cited 2009 Jul 01]; 61(5):615-9. Available from: http:// www.scielo.br/scielo.php?script=sci arttext\&pid=S0034-71672008000500014\&lng=en

24. Mendoza-Parra S, Paravic-Klijn T, Muñoz-Muñoz AM, Barriga OA, Jiménez-Contreras E. Visibility of Latin American nursing research (1959-2005). J Nurs Scholarsh. 2009 Mar; 41(1):54-63.

25. Polit DF, Beck CT. International differences in nursing research, 2005-2006. J Nurs Scholarsh. 2009 Mar; 41(1):44-53.

26. Braga AN. Reflexões sobre a superação do conhecimento fragmentado nos cursos de graduação. In: Leite D, organizador. Pedagogia Universitária: conhecimento, ética e política no ensino superior. Porto Alegre (RS): Universidade Federal do Rio Grande do Sul; 1999.

27. Teixeira E, Vale EG. Tendências e perspectivas do ensino de graduação em enfermagem. In: Vale EG, Fernandes JD, Sordi MRL, organizadores. O ensino de graduação em enfermagem no Brasil. Brasília (DF): INEP/MEC; 2006. p.119-28.

28. Sanjuan QA, Martínez RJR. Nuevo enfoque en el proceso de enseñanza-aprendizaje para la interrelación de conocimientos y formación de clínica/comunitaria. Invest Educ Enferm. 2008; 26 (2 supl):150-9.

29. Medina-Moya, JL, Castillo-Parra, S. La enseñanza de la enfermería como una práctica reflexiva. Texto Contexto Enferm. 2006 Jun; 15(2):303-11.
Correspondência: Marta Lenise do Prado.

Departamento de Enfermagem. Centro de Ciências da Saúde. Universidade Federal de Santa Catarina. 88040-970 - Campus Universitário,Trindade. Florianópolis, SC, Brasil.

E-mail: mpradop@ccs.ufsc.br
Recebido: 14 de setembro de 2010 Aprovação: 14 de dezembro de 2010 